\title{
Dosis de levotiroxina varía según la etiología del hipotiroidismo y el peso corporal
}

\author{
Carlos Alfonso Builes Barrera', Karen Lorena Palacios Bayona², Fabián Alberto Jaimes Barragán ${ }^{3}$
}

\author{
${ }^{1}$ Médico Internista. Endocrinólogo. Hospital Universitario \\ San Vicente Fundación. Profesor de la Facultad de Medicina \\ Universidad de Antioquia \\ ${ }^{2}$ Médica Internista. Endocrinóloga. Universidad de Antioquia \\ ${ }^{3}$ Médico Internista. Doctor en Epidemiologia de las enfermedades \\ infecciosas. Magister en epidemiología clínica. Profesor asociado de \\ la Facultad de Medicina, Universidad de Antioquia.
}

Grupo de Investigación

Grupo de endocrinología clínica y metabolismo, Sección de Endocrinología, Departamento de Medicina Interna. Universidad de Antioquia.

Contacto: endocrinobuiles@gmail.com

\section{Resumen}

Objetivo: Determinar las dosis de levotiroxina necesarias para alcanzar control bioquímico del hipotiroidismo según su etiología, peso corporal, TSH inicial y tiempo desde su diagnóstico.

Población y métodos: Estudio de cohorte retrospectivo en pacientes hipotiroideos mayores de 14 años con control bioquímico de la enfermedad.

Resultados: Se incluyeron 518 pacientes, 90\% mujeres. El hipotiroidismo primario fue la forma más común $(66,3 \%)$, seguido por el hipotiroidismo postiroidectomía total $(13,1 \%)$, poshemitiroidectomía $(6,1 \%)$, central $(5,9 \%)$, posyodo radioactivo $(5,7 \%)$ y postiroiditis subaguda $(2,5 \%)$. Los requerimientos respectivos de levotiroxina $(\mu \mathrm{g} / \mathrm{kg} /$ día) en ese mismo orden fueron: $1,07 \pm 0,48,1,65 \pm 0,46,1,11 \pm 0,52,1,33 \pm$ $0,6,1,51 \pm 0,58$ y $1,11 \pm 0,72(p<0,001)$. La dosis necesaria en pacientes con hipotiroidismo primario se incrementó con el paso del tiempo desde el diagnóstico: menos de 2 años: 0,77 \pm 0,38, entre 2 y 5: 0,90 \pm 0,40, mayor de 5: 1,07 $\pm 0,48(\mathrm{p}<0,001)$.

En pacientes con TSH inicial menor de $10 \mathrm{mUI} / \mathrm{L}$ y con menos de dos años de evolución se normalizó la TSH con dosis de 0,65 $\mu \mathrm{g} \pm 0,33$, mientras aquellos con TSH inicial mayor de 20 necesitaron $1,34 \mu \mathrm{g} \pm 0,68$. $(\mathrm{p}<0,001)$. Se observó una diferencia significativa en las dosis requeridas para lograr control de la enfermedad de acuerdo con el índice de masa corporal, siendo menores por kilo de peso a mayor grado de sobrepeso / obesidad ( $\mathrm{p}=0,0067)$.
Conclusión: La dosis requerida de levotiroxina para alcanzar el control bioquímico depende de la etiología de la enfermedad, de los valores de TSH al momento del diagnóstico, del peso y el tiempo de evolución del hipotiroidismo. La dosis necesaria para el control de formas leves/tempranas es menor que la recomendada en ausencia de función residual.

Palabras clave: hipotiroidismo, dosis, levotiroxina, tratamiento.

\section{Abstract}

Objective: To determine the dose of levothyroxine needed to achieve biochemical control of hypothyroidism based on etiology, body weight, baseline TSH and time since diagnosis.

Population and methods: Retrospective cohort study with hypothyroid patients 14 years of age and older with biochemical control of the disease.

Results: 518 patients were recruited, 90\% of them women. Primary hypothyroidism was the most common form (66.3\%), followed by hypothyroidism post-total thyroidectomy (13.1\%), post hemi-thyroidectomy (6.1\%), central (5.9\%), post-radioactive iodine treatment (5.7\%), and post thyroiditis (2.5\%). The respective levothyroxine requirements $(\mu \mathrm{g} / \mathrm{kg} / \mathrm{d})$ in the same order were: $1.07 \pm 0.48,1.65 \pm 0.46,1.11 \pm 0.52,1.33 \pm 0.6,1.51 \pm 0.58$ and $1.11 \pm 0.72(p<0.001)$. The required dose in patients with primary hypothyroidism increased as a function of time since initial diagnosis: Less than 2 years: $0.77 \pm 0.38,2$ to 5 years: $0.90 \pm 0.40$, more than 5 years: $1.07 \pm 0.48(p<0.001)$.

In patients with baseline TSH levels lower than $10 \mathrm{mIU} / \mathrm{L}$ and less than 2 years of evolution, TSH was normalized with doses of $0.65 \mu \mathrm{g} \pm 0.33$, whereas those with baseline TSH levels higher than 20 needed $1.34 \mu \mathrm{g} \pm 0.68$ ( $p<0.001)$. A significant difference was observed in the dose required to achieve disease control. This difference was related to body mass index, as follows: The greater the degree of overweight/obesity, the lower the doses needed per $\mathrm{kg}$ of body weight ( $p=0.0067)$.

Conclusion: The dose of levothyroxine required to achieve biochemical control depends on the etiology of the disease, TSH levels at the time of diagnosis, weight, and time since onset of hypothyroidism. The dose required to control mild and early forms is lower than the dose recommended in the absence of residual function.

Key words: hypothyroidism, dose levothyroxine, treatment. 


\section{Introducción}

Una aproximación frecuente en el tratamiento del hipotiroidismo primario es calcular la dosis de levotiroxina según el peso corporal, haciendo ajustes progresivos hasta lograr una TSH dentro de rangos poblacionales esperados normales: 0,4 a 3,0 mUI/L en la mayoría de los pacientes adultos y hasta 4,2 mUI/L en adultos mayores ${ }^{(1,2)}$. Varios estudios previos han estimado las necesidades de levotiroxina con base en el peso corporal entre 1,6 y 2,1 $\mu \mathrm{g} / \mathrm{kg} /$ día en ausencia de función tiroidea residual ${ }^{(3-7)}$. En los pacientes con antecedente de cáncer de tiroides que requieren supresión de la TSH, las dosis descritas son de 2,0 a 2,5 $\mu \mathrm{g} / \mathrm{kg} /$ día.

Aunque se ha propuesto que el peso ideal es un mejor predictor de la dosis de levotiroxina entre mujeres pre y posmenopáusicas que el cálculo por el peso actual, no se ha podido demostrar esto para todos los pacientes y la dosis calculada por el peso del paciente es la más aceptada ${ }^{(3,4)}$.

En un estudio realizado en Colombia, la dosis necesaria para el control del hipotiroidismo fue de 1,1 $\mu \mathrm{g} / \mathrm{kg} /$ día de levotiroxina en pacientes con TSH elevada pero menor de 20 $\mathrm{mUI} / \mathrm{L}$, mientras que fue de $1,6 \mu \mathrm{g} / \mathrm{kg} /$ día en aquellos con TSH mayor de $20 \mathrm{mUI} / \mathrm{L}^{(5)}$.

El objetivo de este estudio es determinar las necesidades de levotiroxina para lograr niveles de TSH en metas según la etiología del hipotiroidismo, especialmente en la población con falla tiroidea leve o compensada. También se quiere evaluar si hay diferencias en las necesidades de levotiroxina en pacientes con hipotiroidismo basados en el tiempo de evolución de la enfermedad, el índice de masa corporal y los valores de TSH en el momento del diagnóstico.

\section{Materiales y métodos}

\section{Diseño y población de estudio}

Se diseñó un estudio de cohorte de tipo retrospectivo en el cual se analizaron los pacientes evaluados en la consulta de endocrinología (tiroides) desde el año 2011 hasta el 2013 en dos centros de atención (Hospital Universitario de San Vicente Fundación y consulta privada del médico endocrinólogo Carlos Alfonso Builes Barrera). Se incluyeron pacientes mayores de 14 años, con diagnóstico confirmado de hipotiroidismo de cualquier etiología, con al menos 6 meses de tratamiento médico con levotiroxina y que en el momento de la consulta tuvieran criterios bioquímicos de hipotiroidismo controlado. Se excluyeron las mujeres hipotiroideas en embarazo y aquellos pacientes con tiroidectomía total por cáncer de tiroides que presentaran enfermedad activa con menos de cinco años de la cirugía.

\section{Mediciones}

Para definir hipotiroidismo controlado se estableció un límite inferior de TSH de 0,4 mIU/L y un límite superior de 3,0
$\mathrm{mUI} / \mathrm{L}^{(1)}$. En el caso de pacientes mayores de 60 años se aceptó un límite superior de TSH hasta 4,2 mUI/L $\mathrm{L}^{(2)}$. En pacientes con hipotiroidismo central se definió control bioquímico cuando el valor de la última T4 libre estaba en rangos de normalidad de 0,73 a $1,95 \mathrm{ng} / \mathrm{dL}$.

Los pacientes se clasificaron de acuerdo con el tipo de hipotiroidismo en siete categorías:

1. Hipotiroidismo primario autoinmune con anticuerpos antitiroideos antimicrosomales (antiperoxidasa o antiTPO) y/o antitiroglobulina positivos.

2. Hipotiroidismo primario sin dato de anticuerpos

3. Hipotiroidismo primario postiroiditis subaguda

4. Hipotiroidismo primario posterior a la administración de yodo radioactivo

5. Hipotiroidismo primario posterior a la realización de hemitiroidectomía

6. Hipotiroidismo primario posterior a la realización de tiroidectomía total

7. Hipotiroidismo central.

Se registró la edad, el sexo, el tiempo de diagnóstico del hipotiroidismo, el valor de TSH y/o T4 libre al momento del diagnóstico y de obtener dos controles consecutivos de TSH y/o T4 libre en rango normal, el peso y la talla de los pacientes en la consulta y la dosis de levotiroxina con la que se alcanzó el control bioquímico del hipotiroidismo.

\section{Plan de análisis}

Las variables continuas se describen como promedios con desviación estándar y las variables nominales como proporciones. Las comparaciones entre grupos se hicieron por medio de un análisis de varianza (ANOVA) de una vía o de dos vías, de acuerdo con el número de factores independientes; o de Kruskal-Wallis, según la distribución de los datos. Se consideraron significativos valores de $\mathrm{p}<0,05$.

\section{Resultados}

Se recolectaron los datos de 615 pacientes y 97 se excluyeron del análisis por presentar TSH fuera de las metas establecidas de acuerdo con la edad. El promedio de edad de los 518 pacientes analizados fue de 52,3 $\pm 14,9$ años (rango entre $15 \mathrm{y}$ 88 años), siendo menores de 60 años 337 pacientes $(65,05 \%)$. Con una relación 9 a 1 mujer a hombre, 469 mujeres (90,5\%) y un promedio de tiempo de diagnóstico del hipotiroidismo de $7,23 \pm 7,4$ años. El valor promedio de TSH en el último control médico fue de $1,69 \pm 0,78 \mathrm{mIU} / \mathrm{L}$, mientras que el de T4 libre fue $1,20 \pm 0,31 \mathrm{ng} / \mathrm{dL}$.

Los mayores requerimientos de levotiroxina se produjeron en pacientes postiroidectomía total e hipotiroidismo posyodo radioactivo con 1,6 y $1,5 \mu \mathrm{g} / \mathrm{kg} /$ día, respectivamente, mientras que los menores requerimientos fueron en pacientes con hipotiroidismo primario sin reporte de anticuerpos con 1,03 \pm 
0,46 $\mu \mathrm{g} / \mathrm{kg} /$ día ( $\mathrm{p}<0,001$ para la diferencia entre medias), seguidos de hipotiroidismo poshemitiroidectomía y postiroiditis subaguda como se muestra en la tabla 1.

El valor de la TSH al momento del diagnóstico se obtuvo en 224 de los 518 pacientes estudiados $(43,4 \%)$, siendo en promedio de 22,48 \pm 38,79 mIU/L. Los valores fueron mayores en pacientes con hipotiroidismo posyodo y postiroidectomía (tabla 1).

En el subgrupo de los 344 pacientes con hipotiroidismo primario se encontró asociación directa entre el tiempo de evolución de la enfermedad y una mayor dosis de levotiroxina para alcanzar el control bioquímico (tabla 2).

En el subgrupo de pacientes con hipotiroidismo primario sin registro de anticuerpos antitiroideos se logró el control de
TSH con $0,65 \pm 0,33 \mu \mathrm{g} / \mathrm{kg} /$ día de levotiroxina cuando la TSH inicial fue menor de 10 y con $0,77 \pm 0,002 \mu \mathrm{g} / \mathrm{kg} /$ día de levotiroxina cuando la TSH estuvo entre 10 y 20 mIU/L. En el subgrupo de pacientes con hipotiroidismo primario y anticuerpos antitiroideos positivos documentados se apreció una relación significativa entre el tiempo de diagnóstico de la enfermedad, los valores iniciales de TSH y los requerimientos de levotiroxina necesarios para lograr TSH en metas terapéuticas (tabla 3).

Se observó una diferencia significativa en las dosis requeridas para lograr control de la enfermedad de acuerdo con el índice de masa corporal, siendo menores por kilo de peso a mayor grado de sobrepeso/obesidad ( $\mathrm{p}=0,0067$ ) y mayores necesidades de levotiroxina a mayor tiempo del diagnóstico de hipotiroidismo ( $\mathrm{p}<0,001)$, como se muestra en la tabla 4.

Tabla 1. Requerimiento de levotiroxina según la etiología del hipotiroidismo y el valor inicial de TSH

\begin{tabular}{|c|c|c|c|c|}
\hline $\begin{array}{l}\text { Subtipo de hipotiroidismo } \\
\qquad(\mathrm{N}=\mathbf{5 1 8})\end{array}$ & $\begin{array}{l}N \\
(\%)\end{array}$ & $\begin{array}{l}\text { Edad } \\
\text { (años) }\end{array}$ & $\begin{array}{l}\text { TSH inicial } \\
(\mathbf{n}=\mathbf{2 2 4})\end{array}$ & $\begin{array}{l}\text { Dosis de LT4* } \\
\text { ( } \mu \mathrm{g} / \mathrm{kg} / \mathrm{día})\end{array}$ \\
\hline Primario sin dato de anticuerpos & $264(50,96 \%)$ & $53,9 \pm 14,7$ & $27,56 \pm 48,96(n: 119)$ & $1,03 \pm 0,46$ \\
\hline Primario autoinmune (TPO positivos) & $80(15,41 \%)$ & $47,9 \pm 14,1$ & $12,60 \pm 15,76(n: 53)$ & $1,21 \pm 0,54$ \\
\hline Posterior a tiroidectomía total & $68(13,12 \%)$ & $56,5 \pm 12,1$ & $44 \pm 43,85(n: 27)$ & $1,65 \pm 0,46$ \\
\hline Posterior a hemitiroidectomía & $32(6,17 \%)$ & $55,3 \pm 14,3$ & $31,91 \pm 36,95(n: 5)$ & $1,11 \pm 0,52$ \\
\hline Central & $31(5,98 \%)$ & $51,2 \pm 16,5$ & ----- & $1,33 \pm 0,60$ \\
\hline Posyodo radioactivo & $30(5,79 \%)$ & $41,0 \pm 14,1$ & $63,05 \pm 96,14(n: 10)$ & $1,51 \pm 0,58$ \\
\hline Primario postiroiditis subaguda & $13(2,50 \%)$ & $41,3 \pm 12,3$ & $11,10 \pm 8,04(n: 10)$ & $1,11 \pm 0,72$ \\
\hline
\end{tabular}

Los valores se presentan como promedios \pm desviación estándar. ${ }^{*} \mathrm{p}<0.001$ por prueba de ANOVA

Tabla 2. Relación del tiempo del diagnóstico del hipotiroidismo primario con la dosis requerida para lograr control bioquímico de la enfermedad

\begin{tabular}{c|c|c}
\hline Número de pacientes & Tiempo del diagnóstico (años) & Dosis de levotiroxina ( $\mu \mathrm{g} / \mathbf{k g} / \mathrm{dí}$ )* \\
\hline 59 & $<2$ & $0,77 \pm 0,38$ \\
\hline 100 & $2-5$ & $0,90 \pm 0,40$ \\
\hline 185 & $>5$ & $1,07 \pm 0,48$ \\
\hline
\end{tabular}

${ }^{*} \mathrm{p}<0,001$ por prueba de ANOVA

Tabla 3. Dosis de levotiroxina de acuerdo con valores iniciales de TSH y tiempo desde el diagnóstico en pacientes con hipotiroidismo primario y anticuerpos positivos

\begin{tabular}{c|c|c|c}
\hline \multirow{2}{*}{ Tiempo de diagnóstico } & \multicolumn{3}{|c}{ Valor de TSH (mIU/L) } \\
\cline { 2 - 4 } & $<10(\mathrm{~N}=26)$ & $10-20(\mathrm{~N}=13)$ & $>20(\mathrm{~N}=14)$ \\
\hline 2 2 años $(\mathrm{N}=23)$ & $0,67 \pm 0,15$ & $0,71 \pm 0,31$ & $0,81 \pm 0,38$ \\
\hline$>5$ años $(\mathrm{N}=20)$ & $1,14 \pm 0,61$ & $1,37 \pm 0,60$ & $1,77 \pm 0,24$ \\
\hline
\end{tabular}

Prueba ANOVA de dos vías: 1. Relación tiempo de evolución de la enfermedad con el requerimiento de levotiroxina p < 0,001. 2. Relación valor de TSH al momento del diagnóstico con requerimiento de levotiroxina $p<0,001$. 
Tabla 4. Relación del índice de masa corporal con los requerimientos de levotiroxina y tiempo de evolución del hipotiroidismo en todo el grupo de estudio

\begin{tabular}{|c|c|c|c|c|c|c|}
\hline \multirow{2}{*}{$\begin{array}{l}\text { Años del } \\
\text { diagnóstico }\end{array}$} & \multicolumn{6}{|c|}{ Dosis de levotiroxina de acuerdo con el IMC $\left(\mathrm{Kg} / \mathrm{m}^{2}\right)$ (Número de pacientes) } \\
\hline & $<18,5$ & $18,5-24,9$ & $25-29,9$ & $30-34,9$ & $35-39,9$ & $>40$ \\
\hline$<2$ & & $\begin{array}{c}0,92 \pm 0,54 \\
(n=46)\end{array}$ & $\begin{array}{c}1,09 \pm 0,49 \\
(n=33)\end{array}$ & $\begin{array}{c}1,04 \pm 0,58 \\
(n=9)\end{array}$ & $\begin{array}{c}0,41 \\
(\mathrm{n}=1)\end{array}$ & \\
\hline $2-5$ & $\begin{array}{c}1,79 \pm 0,54 \\
(n=5)\end{array}$ & $\begin{array}{c}1,07 \pm 0,56 \\
(n=68)\end{array}$ & $\begin{array}{c}1,15 \pm 0,48 \\
(n=70)\end{array}$ & $\begin{array}{c}1,06 \pm 0,48 \\
(n=25)\end{array}$ & $\begin{array}{c}1,08 \pm 0,38 \\
(n=7)\end{array}$ & $\begin{array}{c}0,79 \pm 0,17 \\
(n=5)\end{array}$ \\
\hline$>5$ & $\begin{array}{c}2,79 \\
(n=1)\end{array}$ & $\begin{array}{c}1,24 \pm 0,57 \\
(n=125)\end{array}$ & $\begin{array}{c}1,20 \pm 0,42 \\
(n=60)\end{array}$ & $\begin{array}{c}1,17 \pm 0,52 \\
(n=43)\end{array}$ & $\begin{array}{c}1,21 \pm 0,39 \\
(n=13)\end{array}$ & $\begin{array}{c}1,18 \pm 0,74 \\
(n=7)\end{array}$ \\
\hline
\end{tabular}

Prueba ANOVA de dos vías: 1 . Relación tiempo de evolución de la enfermedad con requerimiento de levotiroxina p < 0,001. 2. Relación índice de masa corporal con requerimiento de levotiroxina $\mathrm{p}=0,0067$.

\section{Discusión}

En el presente análisis encontramos que la causa del hipotiroidismo, el tiempo de evolución de la enfermedad, el índice de masa corporal y la TSH inicial determinan la dosis de levotiroxina necesaria para alcanzar el control bioquímico del paciente. Al hacer la estratificación por subgrupos de acuerdo con la etiología del hipotiroidismo, se encuentran los mayores requerimientos en las formas ablativas (cirugía total o terapia con yodo radioactivo), seguidas por la tiroiditis crónica (autoinmune). Lo anterior se relaciona también con un valor de TSH más alto pretratamiento, como reflejo del daño inicial más pronunciado sobre la glándula tiroides, además del deterioro progresivo sobre la función residual en las formas autoinmunes, que se correlaciona con el aumento de la dosis de levotiroxina requerida para el control con el paso de los años a partir del diagnóstico.

Debe destacarse que la gran mayoría de los pacientes con hipotiroidismo primario cursan con títulos positivos de autoanticuerpos, esto se reporta en cerca del 85\% de los casos. En la cohorte evaluada, esta proporción es muy baja y consideramos que la principal explicación es la falta de una medición rutinaria en todos los pacientes en nuestro medio, especialmente cuando tienen valores muy altos de TSH desde el comienzo, además del costo que representa para el paciente. Por otro lado, la inclusión de pacientes con diagnóstico de muchos años de evolución hizo difícil la búsqueda y hallazgo de la información registrada de manera completa como sucede en los trabajos retrospectivos.

La medición de los antiTPO es útil en la práctica clínica para confirmar la etiología autoinmune de la enfermedad, además de la búsqueda activa de otras patologías que pueden hacer parte de un síndrome poliglandular autoinmune.

En la literatura se ha descrito clásicamente que la dosis de suplencia para hipotiroidismo es de 1,6 $\mu \mathrm{g} / \mathrm{kg} /$ día, recomendación extrapolada de estudios en donde hay ausencia completa de la función tiroidea, como en los casos de hipotiroidismo posterior a tiroidectomía total o a la administración de yodo radioactivo ${ }^{(6-8)}$. Sin embargo, en la práctica clínica se observa que la dosis de suplencia requerida depende de la etiología del hipotiroidismo, puesto que en algunos casos aún hay función residual de la glándula tiroides ${ }^{(6)}$.

Kabadi evaluó la etiología del hipotiroidismo como determinante de la dosis de LT4 requerida para alcanzar el control bioquímico $^{(10)}$, encontrando un requerimiento de dosis menores en hipotiroidismo primario debido a tiroiditis de Hashimoto, idiopático o posterior a tiroiditis por irradiación del cuello y dosis más altas luego de tratamiento con yodo radioactivo o tiroidectomía total o subtotal, lo que está de acuerdo con los hallazgos obtenidos en el presente estudio.

Los datos en la literatura son contradictorios en cuanto al valor de la TSH al momento del diagnóstico y su relación con las dosis de suplencia para alcanzar el control bioquímico de la enfermedad. En otro estudio de Kabadi y colaboradores ${ }^{(11)}$, se encontró que hay correlación entre estas variables al realizar un análisis por regresión logarítmica $(r=0,86 ; p<0,00001)$ o regresión lineal $(r=0,83 ; p<0,00001)$, afirmando que la TSH pretratamiento predice la dosis requerida de levotiroxina para alcanzar el control del perfil tiroideo, datos similares a los obtenidos en nuestro análisis. Estos resultados contrastan con los obtenidos por Devdhar y colaboradores ${ }^{(3)}$ quienes concluyeron que los factores que influían en determinar la dosis óptima de levotiroxina eran el sexo y el índice de masa corporal, pero no la TSH inicial registrada al momento del diagnóstico.

Actualmente, el peso real del paciente es el utilizado para calcular la dosis necesaria de suplencia hormonal en el hipotiroidismo ${ }^{(1,6)}$. Sin embargo, estudios previos han demostrado que el peso seco del paciente puede ser una aproximación más exacta para determinar los requerimientos de levotiroxina, especialmente en pacientes con índice de masa corporal aumentado por obesidad o por mayor masa muscular ${ }^{(4)}$. Jouklaas, 2010, encontró que al usar la fórmula del peso ideal para calcular las dosis de levotiroxina, los requerimientos fueron más altos en las mujeres de todas las edades que en hombres ${ }^{(12)}$. Santini y colaboradores ${ }^{(4)}$ demostraron que las diferencias de las dosis entre ambos sexos son un reflejo del peso seco como resultado de las diferencias en la composición corporal entre 
hombres y mujeres. Esta consideración constituye una limitación de nuestro estudio, en donde la dosis de los pacientes fue calculada por el peso real mas no por el peso seco.

El índice de masa corporal tiene buena correlación con la dosis necesaria de levotiroxina en pacientes hipotiroideos postiroidectomía. El cálculo de la dosis convencional por el peso mostró que de 122 pacientes tratados sólo el 32\% estaban eutiroideos, el 53\% de los pacientes con IMC mayor de 30 estaban sobretratados y $46 \%$ de los pacientes con IMC menor de 25 estaban subdosificados ${ }^{(29)}$. Los hallazgos de este trabajo tienen correlación con los datos encontrados de las menores dosis usadas por kilogramo de peso en pacientes con obesidad. Se propone que la dosis inicial de levotiroxina $(\mu \mathrm{g} / \mathrm{kg} /$ día) según el IMC sea de -0,018*IMC +2,13.

La gran mayoría de los pacientes evaluados se encontraron dentro del peso normal o en sobrepeso y sólo el 2,3\% tuvo IMC mayor de 40 (obesidad mórbida). Guardando una relación similar a la reportada para la población general ${ }^{(9)}$.

Existen variables individuales que afectan la biodisponibilidad de la levotiroxina como son los polimorfismos genéticos en las deyodasas y las diferencias particulares en las proteínas de transporte de hormonas tiroideas ${ }^{(5)}$. Adicionalmente, la presentación de la levotiroxina consumida, el tiempo entre la ingestión del medicamento y el consumo de alimentos, y el uso de medicamentos con potencial interferencia en la efectividad de la levotiroxina son variables que no pudieron ser controladas en el presente estudio retrospectivo.

Aunque la levotiroxina genérica se considera bioequivalente con las presentaciones comerciales, no es intercambiable, por lo que se recomienda tener en cuenta que las preparaciones no sean variadas en la farmacia y que si esto sucede se reevalúe la TSH en 6 a 8 semanas luego del cambio de presentación de la levotiroxina ${ }^{(13)}$. Este factor determinante de variabilidad en el control adecuado de los pacientes no es posible conocerlo por la naturaleza retrospectiva del estudio y la escasez de registros particulares frente a la marca de levotiroxina recibida, especialmente en pacientes institucionales.

Otros factores que afectan la biodisponibilidad de la levotiroxina son la adherencia al tratamiento, las interacciones medicamentosas y las comorbilidades ${ }^{(14)}$. Dentro de las enfermedades concomitantes que modifican los requerimientos de LT4 se encuentran la infección por Helicobacter pylori ${ }^{(15)}$, la gastritis crónica ${ }^{(15)}$, la enfermedad celiaca ${ }^{(5)}$, la cirugía bariátrica $^{(16,17)}$ y la giardiasis ${ }^{(18)}$. Respecto a las interacciones medicamentosas se han descrito en la literatura que interfieren con la absorción de LT4 el tratamiento con inhibidores de bomba de protones $^{(19)}$, colestiramina ${ }^{(20)}$, colesevelam ${ }^{(20)}$, poliestireno sulfonato cálcico ${ }^{(19)}$, sulfato ferroso ${ }^{(21)}$, sucralfato ${ }^{(22)}$, antiáci$\operatorname{dos}^{(23)}$, laxantes ${ }^{(23)}$, suplementos de calcio ${ }^{(24)}$, sevelamer ${ }^{(25,26)}$, raloxifeno $^{(27)}$, orlistat ${ }^{(28)}$, entre otros. Por ser un estudio de naturaleza retrospectiva es difícil conocer todas las variables para el análisis de causas que conllevan a incrementos en la dosis necesaria de levotiroxina, constituyendo otra limitante de los resultados.

\section{Conclusiones}

Múltiples elementos intervienen en el control bioquímico del hipotiroidismo. En la población evaluada se encontró una relación entre la dosis necesaria de levotiroxina para lograr el eutiroidismo y la etiología del hipotiroidismo, el tiempo de evolución de la enfermedad, el índice de masa corporal y la TSH inicial, factores que se deben tener en cuenta para determinar la dosis óptima de levotiroxina. La invitación es a individualizar la dosis necesaria para el tratamiento de los pacientes con diagnóstico reciente de hipotiroidismo, especialmente para aquellos con valores iniciales de TSH menores de $10 \mathrm{mIU} / \mathrm{L}$.

Debido a las limitaciones por ser un estudio observacional, se requieren estudios prospectivos para generalizar los hallazgos obtenidos en nuestro análisis.

\section{Referencias}

1. Garber JR, Cobin RH, Gharib H, Hennessey JV, Klein I, Mechanick JI, et al. Clinical Practice Guidelines for Hypothyroidism in Adults: Co-sponsoredby American Association of Clinical Endocrinologists and the American Thyroid Association. Thyroid. 2012; 22(12):1200-35.

2. Hollowell JG, Staehling NW, Flanders WD, Hannon WH, Gunter EW, Spencer CA, et al. Serum TSH, T(4), and thyroid antibodies in the United States population (1988 to 1994): National Health and Nutrition Examination Survey (NHANES III). J Clin Endocrinol Metab. 2002; 87(2): 489-99.

3. Devdhar M, Drooger R, Pehlivanova M, Singh G, Jonklas J. Levothyroxine replacement doses are affected by gender and weight but not age. Thyroid. 2011; 21 (8): 821-827.

4. Santini F, Pinchera A, Marsili A, Ceccarini G, Castagna MG, Valeriano R, Giannetti M, Taddei D, Centoni R, Scartabelli G, Rago T, Mammoli C, Elisei R, Vitti P. Lean body mass is a major determinant of levothyroxine dosage in the treatment of thyroid diseases. J Clin Endocrinol Metab. 2005; 90:124-127.

5. Escobar I. Dosis de levotiroxina (T4) para el tratamiento del hipotiroidismoprimario. En Sociedad Latinoamericana de tiroides. Libro de resúmenes VII Congreso SLAT, 1997. Página 25, resumen 17.

6. Okosieme 0 . Thyroid hormone replacement current status and challenges. Expert Opin Pharmacother. 2011; 12 (15): 2315-2328.

7. Olubowale 0,Chadwick D, Optimization of thyroxine replacement therapy after total or near total thyroidectomy for bening thyroid disease. Br J Surg. 2006. 93: 57-60. 
8. Jin J, Allemang M, McHenry Ch. Levothyroxine replacement dosage determination after thyroidectomy. Am J Surg. 2013; 205: 360-364.

9. Mapas de la situación nutricional en Colombia. http:// home.wfp.org/stellent/groups/public/documents/liaison_offices/wfp186725.pdf

10. Kabadi UM. Influence of age on optimal daily levothyroxine dosage in patients with primary hypothyroidism grouped according to etiology. South Med J. 1997; 90(9):920-4.

11. Kabadi UM, Kabadi MM. Serum thyrotropin in primary hypothyroidism: a reliable and accurate predictor of optimal daily levothyroxine dose. Endocr Pract. 2001; 7(1): 16-18.

12. Jouklaas J. Sex and age differences in levothyroxine dosage requirement. Endocr Pract. 2010; 16:71-79.

13. Hennesey J. Generics vs name brand L- thyroxine products: ¿interchangeable or still not? J Clin Endocrinol Metab. 2013; 98(2):511-14.

14. Centanni M. Thyroxine treatment: absorption, malabsorption, and novel therapeutic approaches. Endocrine. 2013; 43 (1):8-9.

15. Centanni M, Gargano L, Canettieri G, Viceconti N, Franchi A, Delle Fave G, et al. Thyroxine in goiter, Helicobacter pylori infection, and chronic gastritis. N Engl J Med. 2006; 354(17):1787-95.

16. Fazylov R, Soto E, Cohen S, Merola S. Laparoscopic Rouxen-Y gastric bypass surgery on morbidly obese patients with hypothyroidism. Obes Surg. 2008; 18(6):644-7.

17. Rubio IG, Galrão AL, Santo MA, Zanini AC, Medeiros-Neto G. Levothyroxine absorption in morbidly obese patients before and after Roux-En-Y gastric bypass (RYGB) surgery. Obes Surg. 2012; 22(2):253-8.

18. Radaeli R, Diehl L. Increased levothyroxine requirement in a woman with previously well-controlled hypothyroidism and intestinal giardiasis. Arq Bras Endocrinol Metab;55(1):81-84, Feb. 2011.

19. Sachmechi I, Reich DM, Aninyei M, Wibowo F, Gupta G, Kim PJ. Effect of proton pump inhibitors on serum thyroid- stimulating hormone level in euthyroid patients treated with levothyroxine for hypothyroidism. Endocr Pract 2007; 13 (4): 345-350.

20. Liwanpo L, Hershman JM. Conditions and drugs interfering with thyroxine absorption. Best Pract Res Clin Endocrinol Metab 2009; 23 (6): 781-92.

21. Campbell NR, Hasinoff BB, Stalts H, Rao B, Wong NC. Ferrous sulfate reduces thyroxine efficacy in patients with hypothyroidism. Ann Intern Med.1992; 117(12):1010-3.

22. Sherman SI, Tielens ET, Ladenson PW. Sucralfate causes malabsorption of L-thyroxine. Am J Med. 1994; 96(6):531-5.

23. Mersebach H, Rasmussen AK, Kirkegaard L, Feldt-Rasmussen U. Intestinal adsorption of levothyroxine by antacids and laxatives: case stories and in vitro experiments. Pharmacol Toxicol. 1999; 84(3):107-9.

24. Zamfirescu I, Carlson HE. Absorption of levothyroxine when coadministered with various calcium formulations. Thyroid. 2011; 21(5):483-6.

25. John-Kalarickal J, Pearlman G, Carlson HE. New medications which decrease levothyroxine absorption. Thyroid. 2007; 17(8):763-5.

26. Diskin CJ, Stokes TJ, Dansby LM, Radcliff L, Carter TB. Effect of phosphate binders upon TSH and L-thyroxine dose in patients on thyroid replacement. Int Urol Nephrol. 2007; 39(2):599-602.

27. Garwood CL, Van Schepen KA, McDonough RP, Sullivan AL. Increased thyroid-stimulating hormone levels associated with concomitant administration of levothyroxine and raloxifene. Pharmacotherapy. 2006; 26 (6):881-5.

28. Filippatos TD, Derdemezis CS, Gazi IF, Nakou ES, Mikhailidis DP, Elisaf MS. Orlistat-associated adverse effects and drug interactions: a critical review. Drug Saf. 2008; 31(1):53-65.

29. Ojomo KA, Schneider DF, Reiher AE, Lai N, Schaefer S, Chen $\mathrm{H}$, Sippel RS. Using body mass index to predict optimal thyroid dosing after thyroidectomy. J Am Coll Surg. 2013: 216(3), 454-60. 